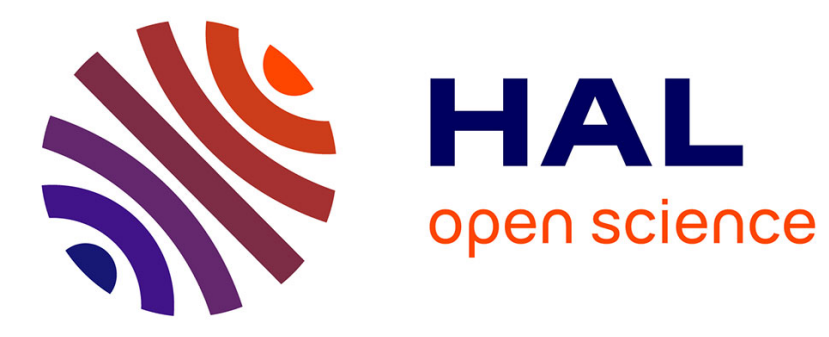

\title{
Tamm plasmon photonic crystals: From bandgap engineering to defect cavity
}

Lydie Ferrier, Hai Son Nguyen, Cécile Jamois, Lotfi Berguiga, Clémentine Symonds, Joël Bellessa, Taha Benyattou

\section{- To cite this version:}

Lydie Ferrier, Hai Son Nguyen, Cécile Jamois, Lotfi Berguiga, Clémentine Symonds, et al.. Tamm plasmon photonic crystals: From bandgap engineering to defect cavity. APL Photonics, 2019, 4 (10), pp.106101. 10.1063/1.5104334 . hal-02353775

\section{HAL Id: hal-02353775 \\ https://hal.science/hal-02353775}

Submitted on 4 Dec 2020

HAL is a multi-disciplinary open access archive for the deposit and dissemination of scientific research documents, whether they are published or not. The documents may come from teaching and research institutions in France or abroad, or from public or private research centers.
L'archive ouverte pluridisciplinaire HAL, est destinée au dépôt et à la diffusion de documents scientifiques de niveau recherche, publiés ou non, émanant des établissements d'enseignement et de recherche français ou étrangers, des laboratoires publics ou privés. 


\section{Tamm plasmon photonic crystals: From bandgap engineering to defect cavity}

Cite as: APL Photonics 4, 106101 (2019); https://doi.org/10.1063/1.5104334

Submitted: 27 April 2019 . Accepted: 09 September 2019 . Published Online: 01 October 2019

(D) Lydie Ferrier, (D) Hai Son Nguyen, Cécile Jamois, (D) Lotfi Berguiga, Clémentine Symonds, Joël Bellessa, and Taha Benyattou

openoccess

\section{ARTICLES YOU MAY BE INTERESTED IN}

Fourier computed tomographic imaging of two dimensional fluorescent objects APL Photonics 4, 106102 (2019); https://doi.org/10.1063/1.5100525

Michelson interferometer modulator based on hybrid silicon and lithium niobate platform APL Photonics 4, 100802 (2019); https://doi.org/10.1063/1.5115136

Spectrometric analysis of silicon nitride films deposited by low-temperature liquid-source CVD

Journal of Applied Physics 126, 133101 (2019); https://doi.org/10.1063/1.5114675

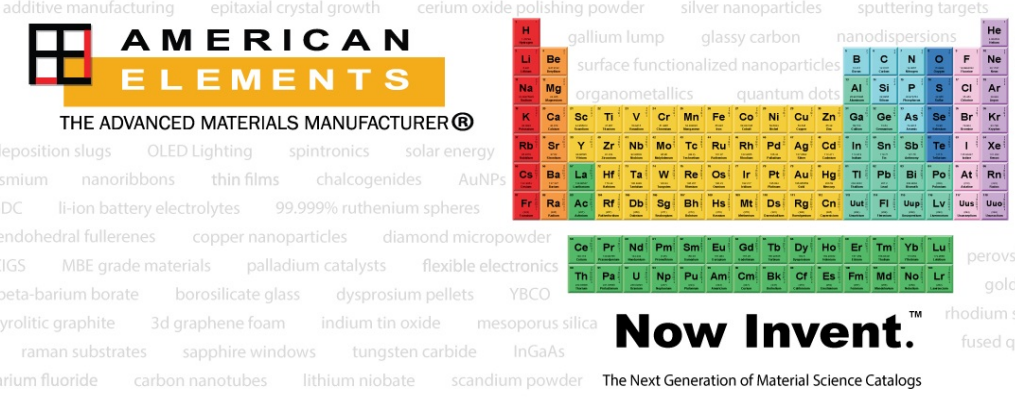

F AMERICAN

THE ADVANCED MATERIALS MANUFACTURER ®

The Next Generation of Material Science Catalogs
Elements opens up a world of possibilities so you can Now Invent

Over 15,000 certified high purity laboratory chemicals, metals, \& advanced materials and a state-of-the-art Research Center. Printable GHS-compliant Safety Data Sheets. Thousands of new products. And much more. All on a secure multi-language "Mobile Responsive" plattor

www.americanelements.com 


\title{
Tamm plasmon photonic crystals: From bandgap engineering to defect cavity
}

\author{
Cite as: APL Photon. 4, 106101 (2019); doi: 10.1063/1.5104334 \\ Submitted: 27 April 2019 • Accepted: 9 September 2019 • \\ Published Online: 1 October 2019
}

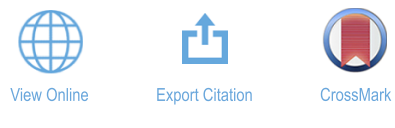

\author{
Lydie Ferrier, ${ }^{1, a)}$ (D) Hai Son Nguyen, ${ }^{2}$ (D) Cécile Jamois, ${ }^{1}$ Lotfi Berguiga, ${ }^{1}$ (D) Clémentine Symonds, ${ }^{3}$ \\ Joël Bellessa, ${ }^{3}$ and Taha Benyattou
}

\begin{abstract}
AFFILIATIONS
${ }^{1}$ Université de Lyon, Institut des Nanotechnologies de Lyon - INL, UMR 5270, INSA Lyon - CNRS, 69621 Villeurbanne, France

${ }^{2}$ Université de Lyon, Institut des Nanotechnologies de Lyon - INL, UMR 5270, Ecole Centrale de Lyon - CNRS, 69134 Ecully, France

${ }^{3}$ Université de Lyon, Institut Lumière Matière - ILM, UMR 5306 Université Lyon 1-CNRS, 69622 Villeurbanne, France
\end{abstract}

a) Electronic mail: Iydie.ferrier@insa-Iyon.fr

\begin{abstract}
Photonic bandgap engineering plays a key role in modern photonics since it allows for an ultimate control of photon propagation in periodic dielectric or metallic media. Tamm plasmon structures are a particularly attractive platform since the electromagnetic field can be completely controlled by patterning the thin metal layer. Up to now, only macroscopic patterning (larger than the operation wavelength) has been experimentally demonstrated, leading to 3D confinement of light but suffering from a lack of fine control of the dispersion properties of Tamm plasmons. Here, we report for the first time the opening of a bandgap in Tamm plasmon structures via subwavelength-periodic patterning of the metallic layer. By adopting a "double period" design, we experimentally put into evidence a photonic bandgap for the TE polarization up to $150 \mathrm{~nm}$ wide in the telecom wavelength range. Moreover, such a design offers a great flexibility to tailor on-demand, and independently, the bandgap size from $30 \mathrm{~nm}$ to $150 \mathrm{~nm}$ and its spectral position within a range of $50 \mathrm{~nm}$. Finally, by implementing a defect cavity within the Tamm plasmon photonic crystal, a $1.6 \mu \mathrm{m}$ cavity supporting a single highly confined Tamm mode is experimentally demonstrated. All experimental results are in perfect agreement with numerical calculations. Our results demonstrate the possibility to engineer novel band dispersion with surface modes of hybrid metallic/dielectric structures, thus opening the way to applications in topological photonics, metamaterials, and parity-time symmetry physics.
\end{abstract}

(C) 2019 Author(s). All article content, except where otherwise noted, is licensed under a Creative Commons Attribution (CC BY) license (http://creativecommons.org/licenses/by/4.0/). https://doi.org/10.1063/1.5104334

\section{INTRODUCTION}

Photonic bandgap engineering plays a key role in modern photonics since it allows for an ultimate control of photon propagation in periodic dielectric or metallic media. ${ }^{1}$ In such structures, photons cannot propagate in the direction of periodicity within given ranges of energies, similarly to the forbidden gaps for electrons in crystals. The simplest example of the apparition of a photonic bandgap in a periodic layered media is the well-known Bragg mirror. ${ }^{2}$ At the edges of the forbidden gap, as the group velocity is close to zero, the high density of states leads to strong enhancement of the lightmatter interaction. This unique property has been widely used for the realization of various nanophotonic devices, with applications such as integrated and mirror-free microlasers ${ }^{3}$ or enhancement of nonlinear effects. ${ }^{4}$ Moreover, the local modification of the periodic pattern of a photonic crystal can induce localized states within the photonic bandgap. This modification can take the form of a line $e^{5,6}$ or a point defect ${ }^{7-9}$ within the periodic modulation, or of a photonic heterostructure, ${ }^{10-12}$ hence creating various guiding structures and cavities with high potential interest. In particular, the concept of photonic nanocavity has been widely used to demonstrate lasing in microcavities, ${ }^{13,14}$ enhancement of spontaneous emission, ${ }^{15,16}$ strong coupling regime between quantum emitters and photonic defect cavity, ${ }^{17,18}$ and exciton polariton lasing in photonic crystal cavities. $^{19}$

Tamm plasmon modes are optical states localized at the interface of a dielectric Bragg mirror and a thin metal layer. ${ }^{20}$ Unlike conventional plasmons, they present much less losses and allow for 
direct coupling to free space modes. ${ }^{21}$ One of the main advantages of Tamm plasmons is the tailoring of the field confinement simply by patterning the thin metal layer, thus avoiding deep etching of nanostructures. Up to now, lateral mode confinement has been achieved by defining micrometric metallic structures such as disks ${ }^{2-25}$ and rectangles, ${ }^{26}$ leading to three-dimensional light confinement that has been experimentally put into evidence by a set of discrete Tamm modes in energy. Brückner et al. ${ }^{27}$ have recently shown that thin metallic periodic arrays with a period of a few microns replicate the parabolic dispersion of Tamm modes in $\mathrm{k}$-space. They have demonstrated that at the edges of the Brillouin zone, where $k= \pm m \pi / a(m$ being an integer and $a$ the period of the grating) the local density of states is greatly enhanced due to the crossing of the replicated dispersions. However, in Brückner's work, the period of the grating is $7.4 \mu \mathrm{m}$, which is more than ten times their mode emission wavelength $(655 \mathrm{~nm})$ : in this regime, the coupling of forward and backward propagating waves at the edge of the Brillouin zone is too small to observe any bandgap opening. The bandgap (if existing) is much smaller than the spectral width of the modes, making it impossible to realize large photonic bandgaps, microcavity Tamm structures, and related band engineering. As a consequence, new metallic nanostructures need to be designed, in order to gain more versatility in the dispersion tailoring of optical Tamm modes and obtain strong light confinement within photonic Tamm cavities.

In this work, we show that a bandgap opening can be observed at the edge of the Brillouin zone, if the coupling strength between forward and backward propagating waves is strongly increased. This is achieved when the period of the lattice is much smaller than the observed mode wavelength. ${ }^{1}$ In particular, we show that a photonic bandgap for TE polarization (electric field along the axis of the metallic stripes) can be opened by patterning the metallic layer of Tamm plasmon structures with a periodic lattice in the photonic crystal regime (i.e., $a / \lambda \ll 1$ ). Moreover, we propose an original design, named "double period," which offers an on-demand control of both the width and the spectral position of the bandgap.
Angle-resolved measurements show perfect agreement with numerical calculations, hence demonstrating that the bandgap width and spectral position can be freely and independently tuned within the telecom range and that a photonic bandgap as large as $150 \mathrm{~nm}$ can be opened around the $1.5 \mu \mathrm{m}$ wavelength. In order to highlight the potential of bandgap engineering, we also introduce lateral confinement of the Tamm modes within the bandgap via a defect cavity of only $1.6 \mu \mathrm{m}$-size. Highly confined states with discrete energy and flat dispersion are observed. These experimental results should open the way to all photonic bandgap applications on a hybrid metaldielectric platform, where the light confinement only relies on metal patterning.

\section{RESULTS AND DISCUSSION}

We first highlight the mechanism of bandgap opening of Tamm plasmon photonic crystals (i.e., Tamm structures with the periodic metallic layer in the regime $a / \lambda \ll 1$ ). Figure 1 (a) shows a schematic view of the photonic Tamm structure, which consists of a 1D periodic gold grating deposited onto a silicon-based Distributed Bragg Reflector (DBR) (consisting of 4 pairs of $\mathrm{Si} / \mathrm{SiO}_{2}$ alternances with $\lambda / 4 n$ layer thickness and center wavelength $\lambda_{\text {Bragg }}=1.5 \mu \mathrm{m}$, leading to a stopband from $1.2 \mu \mathrm{m}$ to $2 \mu \mathrm{m}$ at normal incidence). The energymomentum dispersion of TE-polarized Tamm modes, numerically simulated by the Finite-Difference Time-Domain (FDTD) method, is presented in Fig. 1(b). It clearly shows the backfolding of the Tamm mode induced by the periodic lattice, as well as a complete photonic bandgap in TE polarization at the edge of the first Brillouin zone (i.e., at the $\mathrm{X}$ point with $k_{x}=\pi / a$ ). This bandgap opening results from the diffractive coupling between the forward and backward Tamm modes propagating beneath the metallic layer. A large bandgap width of $120 \mathrm{~nm}$, corresponding to $15 \%$ of the stopband of the DBR, is observed. Such a bandgap width is much larger than the linewidth of Tamm modes $(\sim 10 \mathrm{~nm})$. Figure 1 (c) depicts the electric
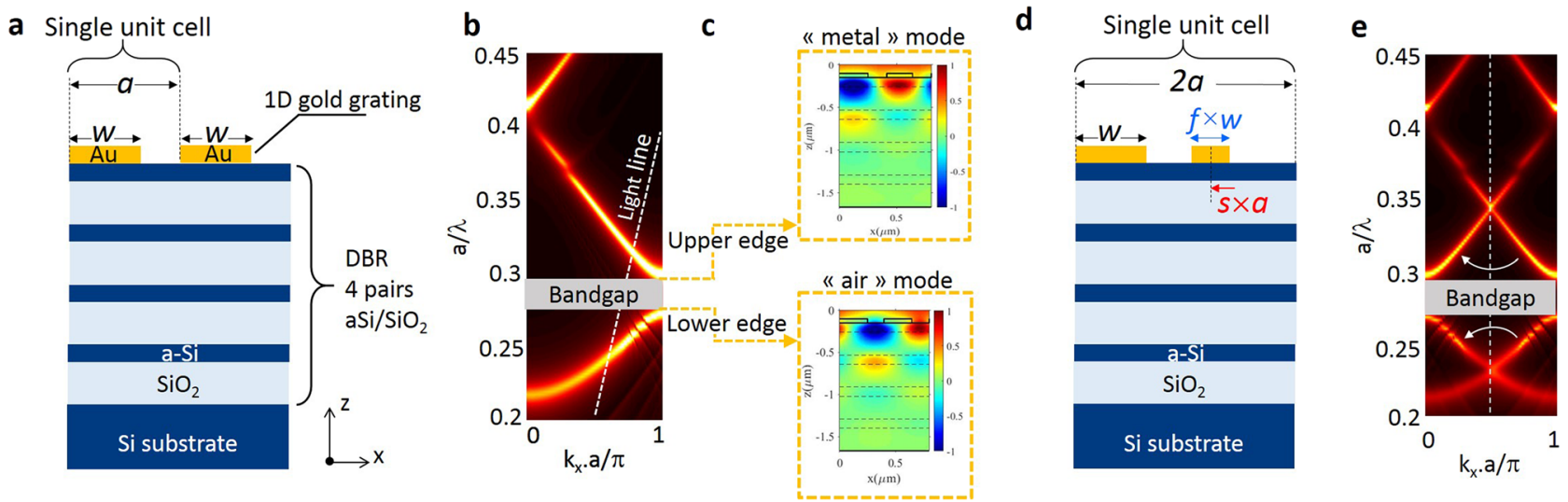

FIG. 1. (a) Sketch of the studied photonic crystal Tamm plasmon structure. (b) Numerical simulation of the energy-momentum dispersion diagram (TE polarization) of a photonic crystal Tamm plasmon with the $50 \mathrm{~nm}$ layer of gold, the period of the pattern is $a=400 \mathrm{~nm}$ and the width of the metal stripes is $w=250 \mathrm{~nm}$. (c) Calculated electric field distribution of two band-edge modes at the $X$ point $\left(k_{x}=\pi / a\right)$. (d) Sketch of the double period design showing the single unit cell containing two gold stripes and the definition of the factor $f$ and shift s. (e) Energy-momentum dispersion diagram (TE polarization) of the double period pattern with $a=400 \mathrm{~nm}, w=250 \mathrm{~nm}, f=0.9$, and $f=0.025$. Band folding is highlighted by the white arrows: band-edge modes at the $X$ point $\left(k_{x}=\pi / a\right)$ are folded to the $\Gamma$-point $\left(k_{x}=0\right)$. 
field distribution at the two band edges. In both cases, we can recognize that the fields show exponential decays from the metal/DBR interface into the Bragg structure, which are characteristic of surface modes such as Tamm modes. ${ }^{25}$ Furthermore, we note that (i) the antinodes of the upper-edge mode are located beneath the metal stripes; thus, we will refer to such localization as "metal" mode, and (ii) the antinodes of the lower-edge mode are located beneath the air grooves. We will refer to such localization as "air" mode. The fact that the "metal" mode is at higher energy than the "air" mode can be explained simply because the real part of the refractive index of metal is lower than for the air.

The results mentioned above demonstrate that a large bandgap can be opened when the metal layer of a Tamm structure is periodically patterned with a subwavelength period. However, the band edges are located below the light line; thus, they are not experimentally accessible for far-field measurements such as reflectivity, transmission, and photoluminescence experiments. Besides, in order to open new degrees of freedom in the tailoring of the band dispersion and increase the spatial and spectral control of the light, the symmetry of the unit cell containing a single gold stripe has to be broken. Thus, in this work, we adopt a "double period" design with two gold stripes per unit cell [see Fig. 1(d)] instead of one as in the initial "single period" [see Fig. 1(a)]. This "double period" design is obtained by shifting and shrinking the second gold stripe with respect to its initial position and size. Quantitatively, the period of the single unit cell is doubled to $2 a$, with the second gold stripe shifted by a distance $s \times a$ and shrunk by a factor $f$, with $0<s, f<1$. This design offers a twofold advantage: (i) Tamm modes at the band edges are now directly accessible from free space and can be probed via far-field experiments. $^{28}$ Indeed, as the unit cell is twice bigger $(2 a)$, the Brillouin zone gets twice smaller: the edge is now located at $k_{x}=\pi / 2 a$ and the band edges initially located below the light line at the $X$-point $\left(k_{x}=\pi / a\right)$ are back-folded to the $\Gamma$-point $\left(k_{x}=0\right)$ [see Fig. $\left.1(\mathrm{e})\right]^{29}$ (ii) The shifting factor $s$ and the shrinking factor $f$ provide efficient degrees of freedom for bandgap engineering. Indeed, by implementing a shifting or a shrinking of the gold stripes, the effective refractive index and the field distribution would be greatly modified, leading to a change of the bandgap size or/and the bandgap position.

Our sample is fabricated on a silicon platform to take advantage of the high refractive index contrast between silica and silicon for the realization of the DBR. As mentioned previously, the DBR is constituted by 4 pairs of $\mathrm{Si} / \mathrm{SiO}_{2}$ layers on a silicon substrate, centered at $1.5 \mu \mathrm{m}$. The microstructure arrays $\left(50 \times 50 \mu \mathrm{m}^{2}\right)$ of $1 \mathrm{D}$ double-period metallic gratings are obtained via a lift-off process: gratings are first defined by electron beam lithography, followed by a $50 \mathrm{~nm}$ gold deposition, and stripping of the underlying resist patterns (see Sec. IV for more details). A scanning electron microscopy (SEM) image of a typical structure is shown in Fig. 2(a), with the inset highlighting the unit cell of the double period design.

The reflectivity spectrum of a structure with $a=400 \mathrm{~nm}$, $w=260 \mathrm{~nm}, s=0.175$, and $f=0.55$ at normal incidence is presented in Fig. 2(b). Two dips corresponding to the two band edges, separated by a bandgap of $130 \mathrm{~nm}$, are experimentally observed, and perfectly reproduced by numerical simulations using the Rigorous Coupled-Wave Analysis (RCWA) method. To demonstrate that these dips correspond to the band edges of two Tamm modes with opposite curvature, we visualize the band diagram in the momentum space by performing angle-resolved microreflectivity measurements (see Sec. IV). The experimental band diagram, shown in the right panel of Fig. 2(c), provides clear evidence of the two band edges at $k_{x}=0$ and of the complete photonic bandgap with $130 \mathrm{~nm}$ width, centered at $1412 \mathrm{~nm}$. These data are perfectly reproduced by RCWA calculations [left panel of Fig. 2(c)]. The small gaps at $k_{x} \neq 0$ of the

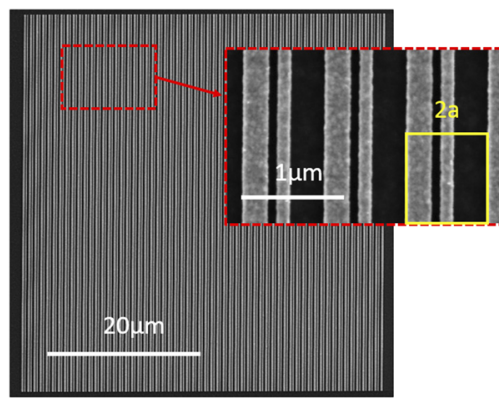

b

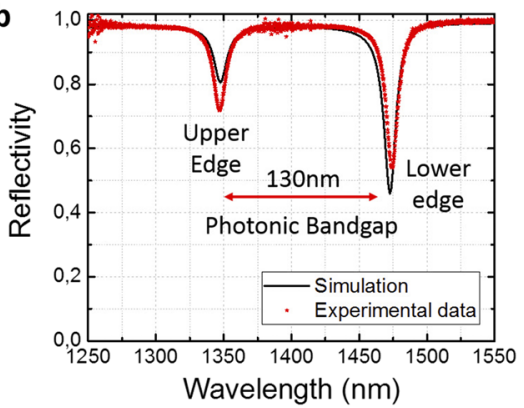

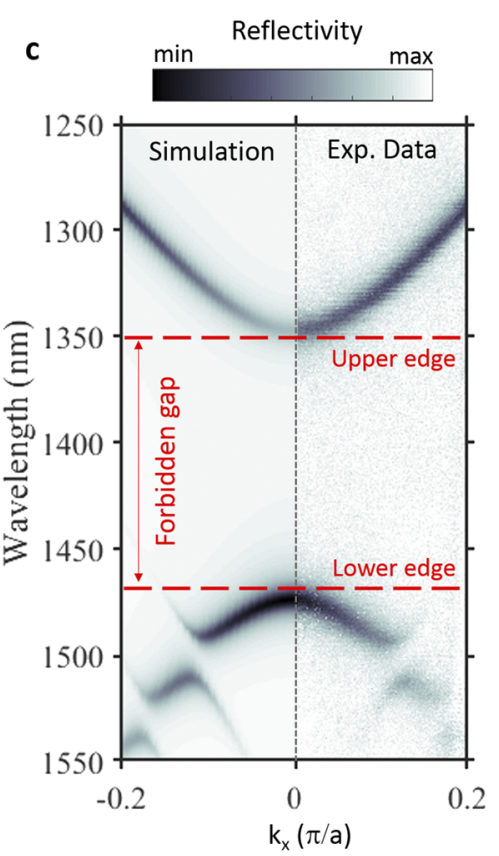

FIG. 2. (a) SEM image of a double period structure. Inset is a zoomed-in image to highlight the structure details on a single unit cell. (b) Reflectivity spectrum at normal incidence of the structure with $a=400 \mathrm{~nm}, w=260 \mathrm{~nm}, s$ $=0.175$, and $f=0.55$. The red dots are experimental data, while the black line is the RCWA calculation. (c) Dispersion diagram obtained by angle-resolved reflectivity spectra. The right (left) panel corresponds to experimental data (RCWA calculation). 
lower band are due to the coupling between Tamm Bloch modes with the propagating mode within the Bragg mirror. ${ }^{30}$ In the following, we will show how such a bandgap can be tailored on-demand by playing with the shifting factor $s$ and the shrinking factor $f$.

\section{A. Bandgap-size tuning}

Figure 3(a) reports experimental measurements and RCWA calculations of the band edge positions when varying the shifting factor $s$ while keeping the shrinking factor at the fixed value $f=0.55$. The band edge positions correspond to the two reflection dips as shown in Fig. 1(b). For the smallest shifting factor $s$, the structure perturbation is too small to experimentally observe the lower edge mode in the reflection spectrum. These results clearly show that a simple variation of the shifting factor enables us to tune the bandgap width from $30 \mathrm{~nm}$ to $150 \mathrm{~nm}$ (i.e., $500 \%$ of variation) without any change of its central wavelength. In this case, the amount of metal (or filling factor) is the same when the shifting factor $s$ is increased: as a result, the central wavelength of the bandgap is fixed since the effective index of each band edge mode remains the same. The effect of bandgap-size tuning is explained by a confinement reduction of the upper edge mode, together with a confinement enhancement of the lower edge state when increasing $s$. In order to illustrate this phenomenon, the electric field distribution of each band edge mode is presented in Fig. 3(b) for two extreme values of the shifting factor $(s=0.025$ and $s=0.4)$. It shows that for $s=0.025$, the field distribution of each mode is almost the same as in the case of the simple period design [Fig. 1(c)]: the upper edge is a "metal" mode with the field confined beneath the metal stripes and the lower edge is an "air" mode with the field confined beneath the air grooves. However, for $s=0.4$, a large part of the field of the upper edge is pushed beneath the air grooves, and inversely, a large part of the field of the lower edge is pushed beneath the metal stripes. As a consequence, the confinement of the upper edge mode is reduced, while the one of the lower edge modes is enhanced.

\section{B. Bandgap-center tuning}

Figure 3(c) reports experimental measurements and RCWA calculations of the band edge positions when varying the shrinking
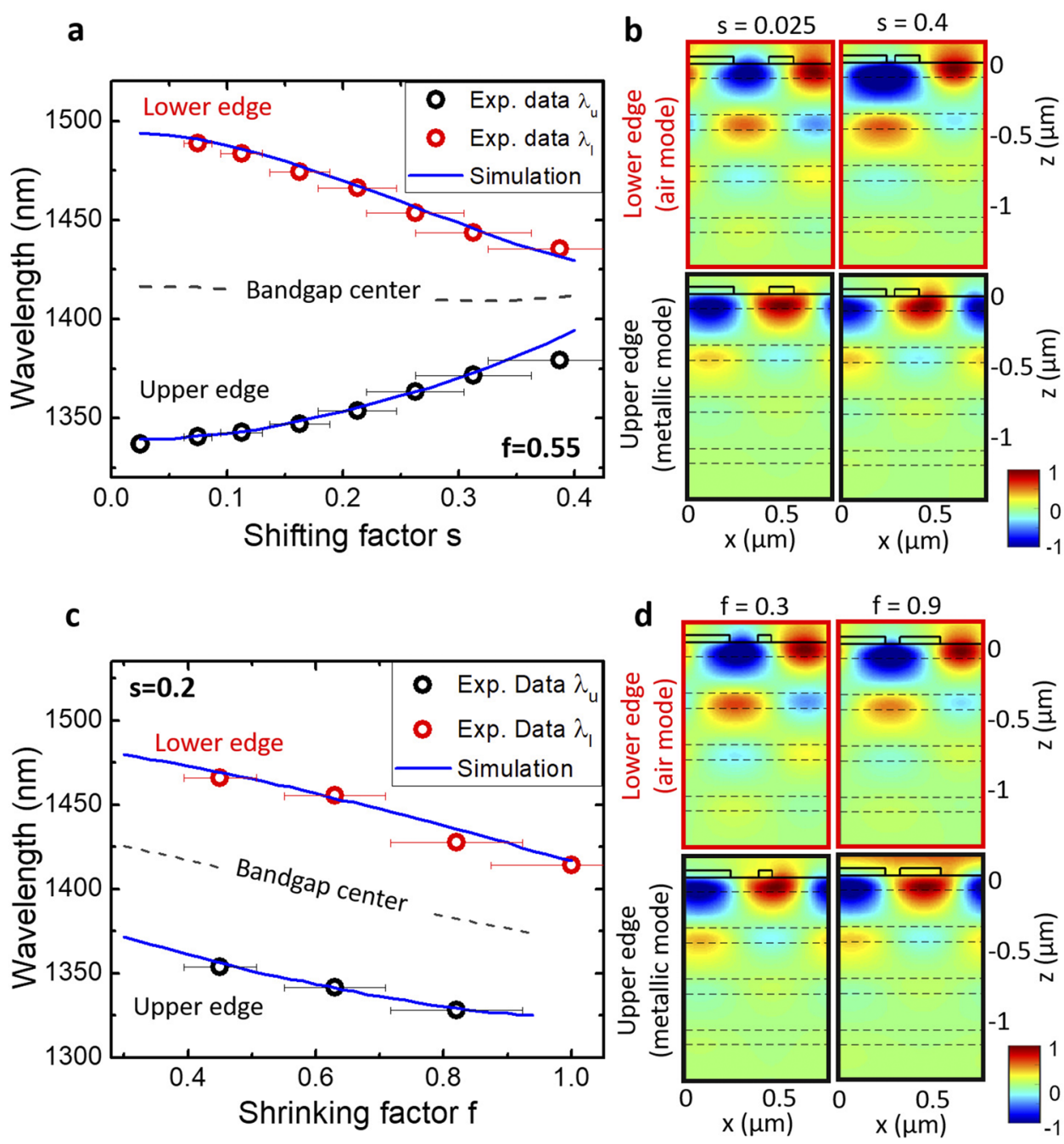

FIG. 3. Bandgap tailoring by tuning the double period design parameters $s$ (shift) and fraction $f[(a)$ and $(c)]$. Variation of band edge positions while varying (a) the shifting factor with constant shrinking factor $f=0.55$ and (c) the shrinking factor with constant shifting parameter $s$ $=0.2$. The blue line shows the results of RCWA calculation. [(b) and (d)] Electric field distributions corresponding to extreme cases of (a) and (c). 
factor $f$, while keeping the shifting factor at the fixed value $s=$ 0.2 . In contrast to the previous observation, these results show that the bandgap width remains almost constant (i.e., $\sim 120 \mathrm{~nm}$ ), whereas its central wavelength can be freely tuned within $50 \mathrm{~nm}$ (i.e., $42 \%$ of the bandgap), simply by changing the shrinking factor. This effect is explained by the confinement enhancement of both band-edge modes when decreasing the shrinking parameter $f$. In order to illustrate this phenomenon, the electric field distribution of each band-edge mode is presented in Fig. 3(d) for two extreme values of the shrinking factor $(f=0.3$ and $f=0.9$ ). On one hand, for both case, the upper-edge is always a "metal" mode and the lower-edge is always an "air" mode. This probably explains why the bandgap width remains constant for different values of $f$. On the other hand, when the second gold stripe is shrunk, there is more air space for both modes to leak, leading to a confinement reduction for both of them. This explains the redshift of the bandgap center when decreasing $f$ (i.e., increasing the shrinking). Finally, in order to further highlight the potential of bandgap engineering offered by photonic crystal Tamm structures, we implement a defect cavity within a photonic heterostructure. ${ }^{10-12}$ These heterostructures are obtained by introducing a local geometrical modification (i.e., defect) in the lattice parameters of a photonic crystal to create a "photonic crystal defect" supporting modes at specific wavelengths that lie within the bandgap of the surrounding lattice (i.e., cladding region). This results in light confinement in the heterostructure cavity. ${ }^{11}$ In our heterostructure design, we consider a cladding structure with parameters $a=400 \mathrm{~nm}, w$ $=250 \mathrm{~nm}, s=0.15$, and $f=0.5$. The defect cavity has exactly the same parameters as the cladding, except for the shrinking factor $f=1$.
According to the results shown in Fig. 3(c), the bandgap size of both the cladding region and the defect is the same, but the bandgap center of the defect is red shifted with respect to the one of the cladding. This leads to the confinement of the lower edge mode within the bandgap of the cladding structure. To experimentally demonstrate such confinement, three cavities of different sizes have been fabricated. The corresponding cavity sizes are 2 unit cells $(1.6 \mu \mathrm{m})$, 7 unit cells $(5.6 \mu \mathrm{m})$, and 12 unit cells $(9.6 \mu \mathrm{m})$. Figure $4(\mathrm{a})$ presents SEM images of the 2-unit-cells cavity, illustrating the fabrication quality of our samples. Angle-resolved microreflectivity experiments have been performed on each cavity, as well as on the cladding for comparison. The dispersion diagrams obtained from the experimental measurements are reported in Figs. 4(b) and 4(c) for the cavities and the cladding, respectively. Comparing with the measurements of Fig. 4(c) performed on the cladding, where the photonic bandgap extends from $1350 \mathrm{~nm}$ to $1500 \mathrm{~nm}$, the cavity measurements all show discrete modes within the corresponding wavelength range, hence demonstrating the apparition of discrete Tamm modes inside the cladding bandgap for all cavities, with increasing mode spacing when reducing the cavity size as highlighted in Fig. 4(b). In the case of the $5.6 \mu \mathrm{m}$ cavity, the mode spacing is $19 \mathrm{~nm}$ between the first $(1440 \mathrm{~nm})$ and second $(1459 \mathrm{~nm})$ modes, and $23 \mathrm{~nm}$ between the second and third modes, respectively; considering the $9.6 \mu \mathrm{m}$ cavity, the mode spacing is reduced to $11 \mathrm{~nm}$ between the first $(1436 \mathrm{~nm})$ and second $(1450 \mathrm{~nm})$ modes, and to $10 \mathrm{~nm}$ between the second and third modes, respectively. Interestingly, unlike the case of confinement induced by metallic patches ${ }^{22,23}$ where the initial Tamm mode has positive effective mass, the Tamm mode that is confined within our cavities is the lower edge mode with a

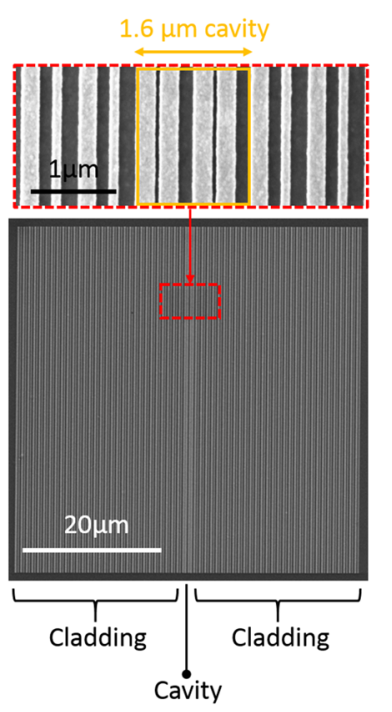

b

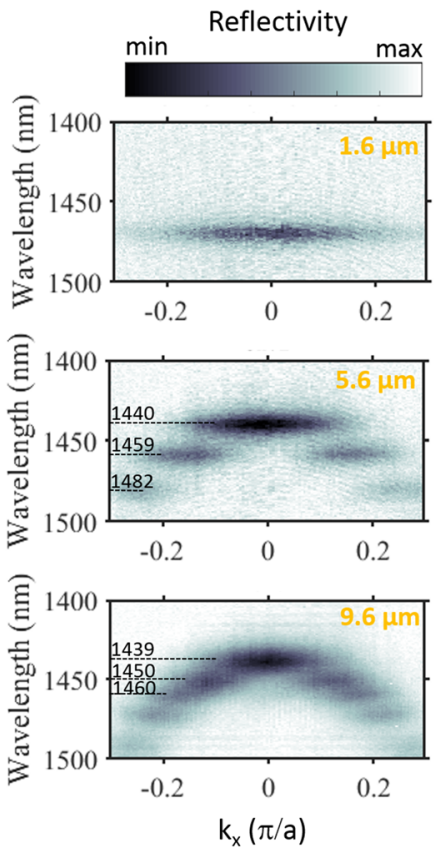

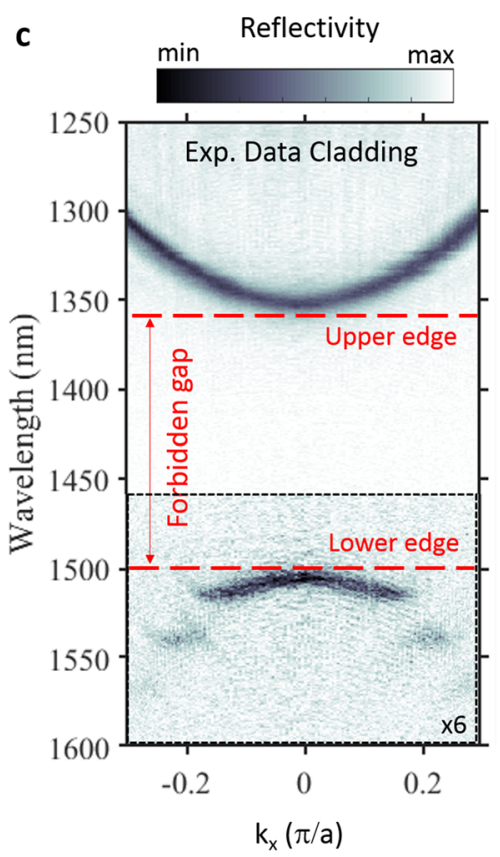

FIG. 4. Experimental demonstration of a photonic Tamm heterostructure cavity. (a) SEM images of the 2 unit cell cavity. [(b) and (c)] Experimental measurements of the angle-resolved microreflectivity experiment performing on (b) three cavities of size of 2 unit cells $(1.6 \mu \mathrm{m}), 7$ unit cells $(5.6 \mu \mathrm{m})$, and 12 unit cells $(9.6 \mu \mathrm{m})$ and $(\mathrm{c})$ the cladding region of the cavities. 
negative effective mass. That explains why the fundamental mode of our cavities is the mode with highest energy. It should also be highlighted that in the case of the smallest cavity (i.e., size of 2 unit cells), the confinement is strong enough to push away higher-order modes outside the cladding bandgap so that only the fundamental mode is observed.

In an analogous way to the study of electron and hole confinement in solid-state heterostructures, the confined Tamm modes can a

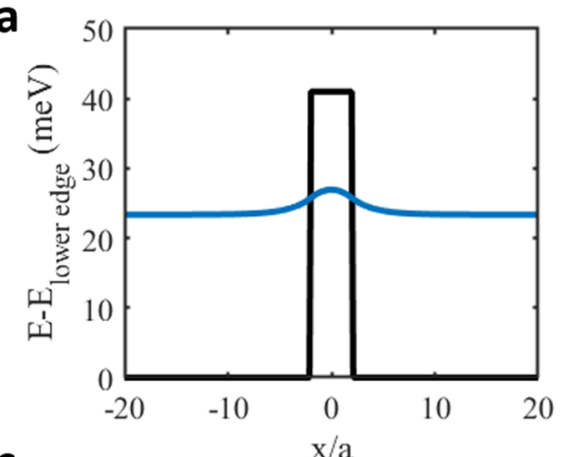

C
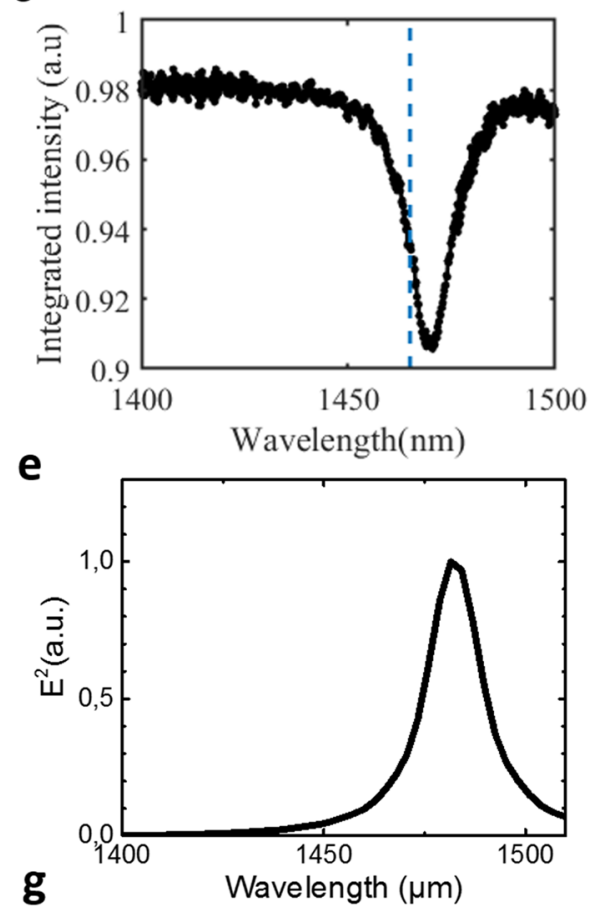

b

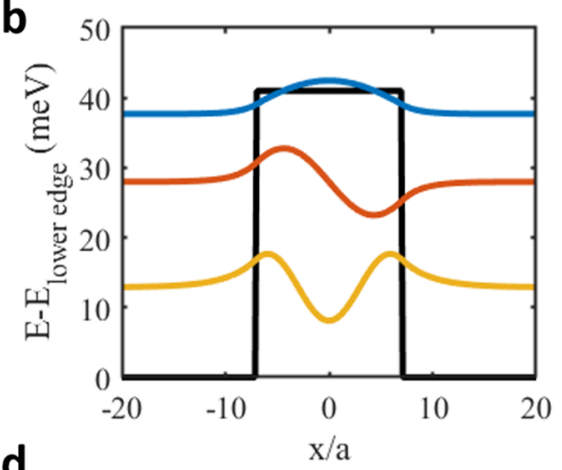

d

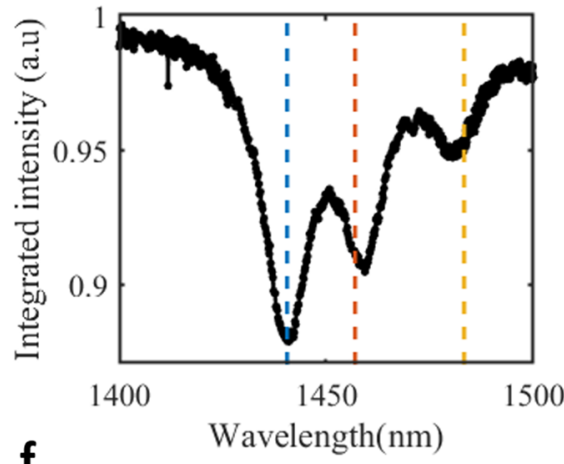

f
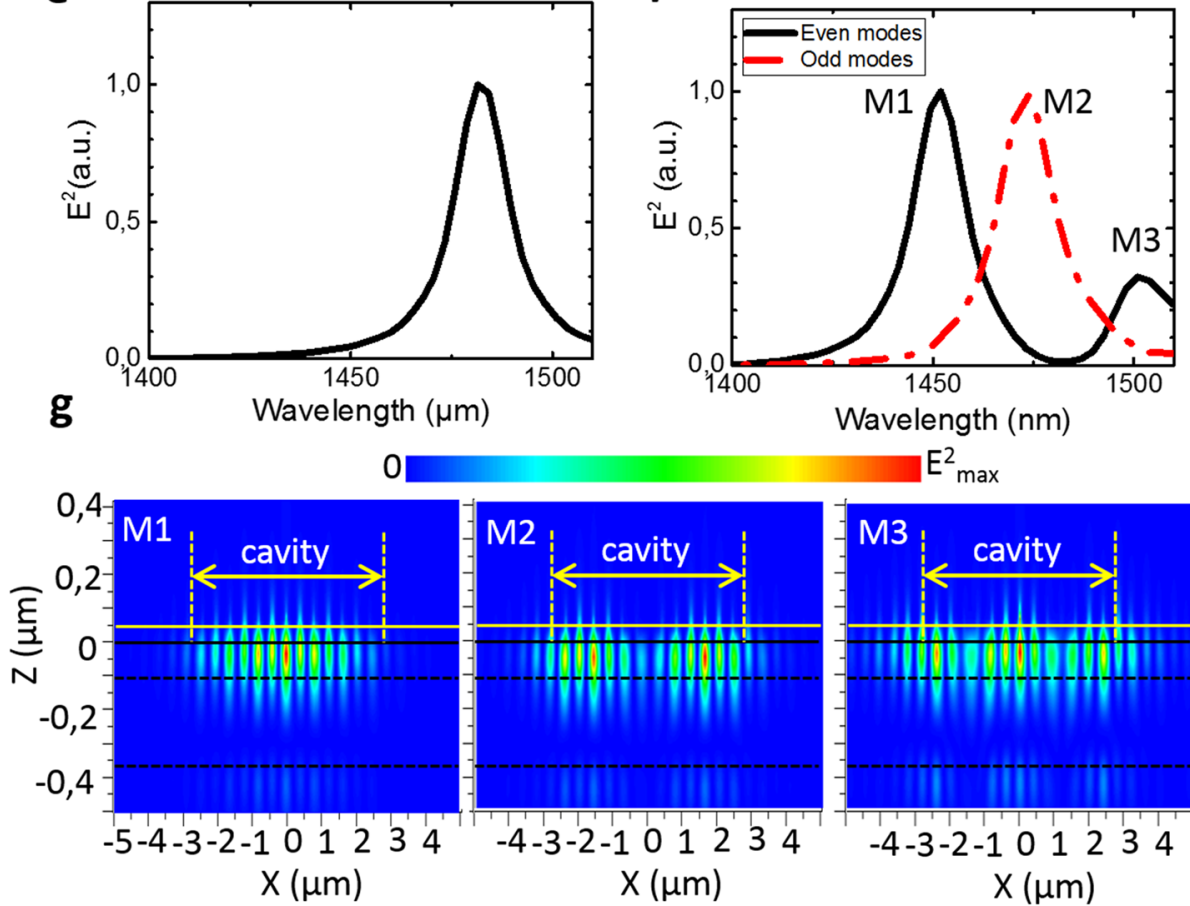

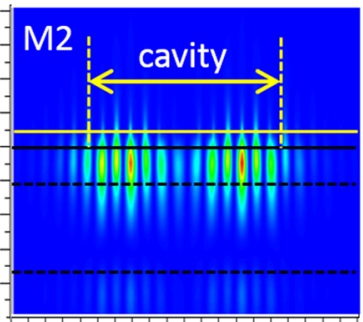

$-4-3-2-1 \quad 0 \quad 1 \quad 2 \quad 3 \quad 4$ $\mathrm{X}(\mu \mathrm{m})$

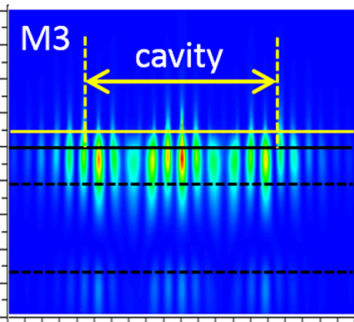

$-4-3-2-1012345$ $X(\mu \mathrm{m})$
FIG. 5. Envelope functions calculated by solving the Schrödinger equation for $1.6 \mu \mathrm{m}$ (a) and $5.6 \mu \mathrm{m}$-size (b) cavities showing a single confined mode for the $1.6 \mu \mathrm{m}$-size cavity and three confined modes for the $5.6 \mu \mathrm{m}$ cavity. (c) Integrated intensity over all angles of the reflectivity signal for the $1.6 \mu \mathrm{m}$-size cavity and (d) $5.6 \mu \mathrm{m}$-size cavity. Eigenvalues of the Schrödinger equation for each cavity are represented by vertical dashed lines. FDTD simulation results for the (e) $1.6 \mu \mathrm{m}$-size cavity and (f) $5.6 \mu \mathrm{m}$-size cavity showing one and three confined modes, respectively. $(\mathrm{g})$ Electric field maps for the first three confined cavity states of the $5.6 \mu \mathrm{m}$-size cavity. Only the $x$-boundaries of the cavity are shown for sake of clarity (and not the detailed Au grating). 
be numerically calculated by solving the Schrödinger equation of the envelope function $\Psi(x)$,

$$
-\frac{\hbar^{2}}{2 m^{*}} \frac{d^{2} \Psi(x)}{d x^{2}}+V(x) \Psi(x)=E \Psi(x),
$$

where $m^{*}<0$ is the photonic effective mass of the lower edge mode and $V(x)$ is the confinement potential of this mode due to the heterostructure landscape: $V(x)=V_{0}>0$ in the cavity and $V(x)=0$ in the cladding. We extract $m^{*} \approx-2.4 \times 10^{-5} m_{\text {electron }}$ by fitting the dispersion of the lower edge mode in the cladding with the parabolic approximation in the vicinity of the $\Gamma$-point. We estimate $V_{0} \approx 41 \mathrm{meV}$, corresponding to the blueshift of the quasicontinuous dispersion of the biggest cavity $(9.6 \mu \mathrm{m})$ with respect to the lower edge mode in the cladding. The envelop functions, numerically calculated by solving the Schrödinger equation for $1.6 \mu \mathrm{m}$-size cavity and $5.6 \mu \mathrm{m}$-size cavity, are depicted in Figs. 5(a) and 5(b), respectively. Qualitatively, good agreement with the experimental results is observed: the $1.6 \mu \mathrm{m}$-size cavity is single mode, while the $5.6 \mu \mathrm{m}$-size cavity supports three confined modes. Figures 5(c) and 5(d) present the integrated intensity over all angles of the reflectivity signal of the $1.6 \mu \mathrm{m}$-size cavity and the $5.6 \mu \mathrm{m}$-size cavity, respectively. The cavity modes are identified as the reflectivity dips in these spectra. The eigenvalues calculated by numerical resolution of the Schrödinger equation are also presented in the same figures (vertical dashed lines), showing a very good spectral agreement with the experimental measurements.

We also calculated direct mode shapes and resonant wavelengths for these cavity structures by using an electromagnetic numerical method. Thus, we performed $2 \mathrm{D}$ finite difference in the time domain (FDTD) simulations to calculate the position of the confined states in the cavity. We excited the structure using a single electrical dipole at the center of the cavity to compute the symmetric modes. For the antisymmetric modes, we used a pair of dipoles with a $\pi$ phase difference located symmetrically with respect to the center of the cavity. Qualitatively, good agreement with experimental results is observed: the $1.6 \mu \mathrm{m}$-size cavity is single mode, while for the $5.6 \mu \mathrm{m}$-size, cavity three peaks are obtained, identified by their parity: 2 even modes and 1 odd mode [Figs. 5(e) and 5(f)]. The corresponding electric field maps of Fig. 5(g) clearly show that the first two cavity states are well localized within the cavity, whereas the third one extends to the cladding. This can be explained by the spectral localization of this state very close to the band edge of the cladding. These results clearly show that by using a Tamm photonic crystal, it is possible to design efficient cavity heterostructures.

\section{CONCLUSION}

In conclusion, the present work demonstrates band diagram engineering of optical Tamm modes for the first time, by periodically patterning the metal layer in the photonic crystal regime $(a / \lambda \ll 1)$. By adopting a "double period" design, we have experimentally put into evidence a TE photonic bandgap up to $150 \mathrm{~nm}$ wide in the telecom wavelength range. Moreover, we have also shown that our design makes it possible to tailor on-demand, and independently, the bandgap size and its spectral position. Experi- mental results highlight a continuous tuning of the bandgap size from $30 \mathrm{~nm}$ to $150 \mathrm{~nm}$ and of the bandgap center within a $50 \mathrm{~nm}$ range. All of our experimental data are perfectly reproduced by numerical calculations. Moreover, by introducing a defect cavity within our Tamm plasmon photonic crystal, a heterostructure cavity of $1.6 \mu \mathrm{m}$ supporting a single highly confined Tamm mode is experimentally demonstrated. It should be pointed out that all $1 \mathrm{D}$ photonic crystal concepts developed in this work can be generalized to the case of $2 \mathrm{D}$ photonic crystals, with the possibility of even more degrees of freedom for bandgap engineering. Our results open exciting perspectives, since novel band engineering should also be possible in such hybrid metal-dielectric structures, in views of observing properties such as Dirac cones ${ }^{28,32-34}$ or flat bands ${ }^{28,35,36}$ with surface modes. This should pave the way toward contemporary topics of optics research such as topological photonics, ${ }^{37}$ metamaterials, ${ }^{32,33,38,39}$ coupling with $2 \mathrm{D}$ materials, ${ }^{40,41}$ non-Hermitian physics, and parity-time symmetry. ${ }^{34,42,43}$

\section{METHODS}

\section{A. Sample fabrication}

The bottom DBR consists of 4 pairs of amorphous $\mathrm{Si} / \mathrm{SiO}_{2}$ $\lambda / 4 \mathrm{n}$ layers grown on a silicon substrate. It has been externally fabricated using a combination of PECVD and high density plasma chemical vapor (HDPCVD), and characterized via complementary techniques such as ellipsometry of the individual layers, reflectivity, and SEM measurements. The targeted central wavelength of the mirror stopband is $1.55 \mu \mathrm{m}$, corresponding to a-Si and $\mathrm{SiO}_{2}$ thicknesses of $110 \mathrm{~nm}$ and $267 \mathrm{~nm}$, respectively. Using these parameters, the photonic stopband ranges from $1.2 \mu \mathrm{m}$ to $2 \mu \mathrm{m}$. The periodic metallic patterns are defined using electron beam lithography followed by a $50 \mathrm{~nm}$ gold deposition. Electron beam lithography is performed on a modified SEM [field emission gun (FEG)- Inspect F e-beam lithography system and Raith Elphy Plus is used for the writing]. The $50 \mathrm{~nm}$ gold layer is evaporated using e-beam evaporation after development of the e-beam resists. A liftoff with acetone is performed at the end of the process. The period of the 1D metallic gratings is chosen to obtain a back-folded Tamm Bloch mode at the $\Gamma$-point between $1.3 \mu \mathrm{m}$ and $1.5 \mu \mathrm{m}(a=400 \mathrm{~nm})$. The size of each array is $50 \mu \mathrm{m} \times 50 \mu \mathrm{m}$.

\section{B. Micro reflectivity measurements}

Microreflectivity experiments are performed at room temperature using a wide spectral white light source (halogen lamp). The Fourier space imaging principle is based on the following procedure: ${ }^{44}$ the samples are excited at normal incidence through a high aperture microscope objective (the use of a high aperture microscope objective allows for the excitation of a range of angles around the normal incidence from $-24.8^{\circ}$ to $24.8^{\circ}$ for bandgap measurements by using a microscope objective with $\mathrm{NA}=0.42$ ). The reflected light by the patterns is collected through the same objective. By using a set of optical lenses, we can image either the real space (i.e., near field imaging) or Fourier space (i.e., far field imaging) at the entrance slit of the spectrometer. Imaging is realized by an infrared camera (InGaAs). A half waveplate and a polarizer are placed in the path of collected light to retrieve only the TE polarization (parallel to the grating structures). 


\section{SUPPLEMENTARY MATERIAL}

See the supplementary material for more information about the origin of the observed modes.

\section{ACKNOWLEDGMENTS}

The authors would like to thank the staff at the NanoLyon Technological Platform for their help and support in all nanofabrication processes. L.F. acknowledges E. Drouard for useful discussions on numerical simulations. The authors gratefully acknowledge financial support from the French National Research Agency (ANR) under the project NEHMESIS (Grant No. ANR-13BS10-0004).

\section{REFERENCES}

${ }^{1}$ J. D. Joannopoulos, P. R. Villeneuve, and S. Fan, "Photonic crystals: Putting a new twist on light," Nature 386, 143-149 (1997).

${ }^{2}$ P. Yeh, Phys. Today 61, 146 (2005); Optical Waves in Layered Media (Wiley, New York, 2005), Vol. 95.

${ }^{3}$ H.-Y. Ryu, S.-H. Kwon, Y.-J. Lee, Y.-H. Lee, and J.-S. Kim, "Very-low-threshold photonic band-edge lasers from free-standing triangular photonic crystal slabs," Appl. Phys. Lett. 80, 3476-3478 (2002).

${ }^{4}$ C. Monat, B. Corcoran, M. Ebnali-Heidari, C. Grillet, B. J. Eggleton, T. P. White, L. O'Faolain, and T. F. Krauss, "Slow light enhancement of nonlinear effects in silicon engineered photonic crystal waveguides," Opt. Express 17, 2944 (2009).

${ }^{5}$ J. S. Foresi, P. R. Villeneuve, J. Ferrera, E. R. Thoen, G. Steinmeyer, S. Fan, J. D. Joannopoulos, L. C. Kimerling, H. I. Smith, and E. P. Ippen, "Photonic-bandgap microcavities in optical waveguides," Nature 390, 143-145 (1997).

${ }^{6}$ M. V. Kotlyar, T. Karle, M. D. Settle, L. O’Faolain, and T. F. Krauss, "Lowloss photonic crystal defect waveguides in InP,” Appl. Phys. Lett. 84, 3588-3590 (2004).

${ }^{7}$ O. Painter, J. Vučković, and A. Scherer, "Defect modes of a two-dimensional photonic crystal in an optically thin dielectric slab," J. Opt. Soc. Am. B 16, 275-285 (1999).

${ }^{8}$ M. Qi, E. Lidorikis, P. T. Rakich, S. G. Johnson, J. D. Joannopoulos, E. P. Ippen, and H. I. Smith, "A three-dimensional optical photonic crystal with designed point defects," Nature 429, 538-542 (2004).

${ }^{9}$ Y. Akahane, T. Asano, B. S. Song, and S. Noda, "High-Q photonic nanocavity in a two-dimensional photonic crystal," Nature 425, 944-947 (2003); e-print arXiv:1609.05510.

${ }^{10}$ B. S. Song, S. Noda, T. Asano, and Y. Akahane, "Ultra-high-Q photonic doubleheterostructure nanocavity," Nat. Mater. 4, 207-210 (2005).

${ }^{11}$ E. Istrate and E. H. Sargent, "Photonic crystal heterostructures and interfaces," Rev. Mod. Phys. 78, 455-481 (2006).

${ }^{12}$ L. Ferrier, P. Rojo-Romeo, E. Drouard, X. Letatre, and P. Viktorovitch, "Slow Bloch mode confinement in 2D photonic crystals for surface operating devices," Opt. Express 16, 3136-3145 (2008).

${ }^{13}$ O. Painter, R. K. Lee, A. Scherer, A. Yariv, J. D. O’Brien, P. D. Dapkus, and I. Kim, "Two-dimensional photonic band-gap defect mode laser," Science 284, 1819-1821 (1999).

${ }^{14} \mathrm{H}$. Altug, D. Englund, and J. Vučković, "Ultrafast photonic crystal nanocavity laser," Nat. Phys. 2, 484-488 (2006); e-print arXiv:0908.3559.

${ }^{15}$ M. Boroditsky, R. Vrijen, T. F. Krauss, R. Coccioli, R. Bhat, and E. Yablonovitch, "Spontaneous emission extraction and Purcell enhancement from thin-film 2-D photonic crystals," J. Lightwave Technol. 17, 2096-2112 (1999).

${ }^{16}$ D. Englund, D. Fattal, E. Waks, G. Solomon, B. Zhang, T. Nakaoka, Y. Arakawa, Y. Yamamoto, and J. Vučković, "Controlling the spontaneous emission rate of single quantum dots in a two-dimensional photonic crystal," Phys. Rev. Lett. 95, 013904 (2005); e-print arXiv:0501091 [quant-ph].

${ }^{17}$ A. Christ, S. G. Tikhodeev, N. A. Gippius, J. Kuhl, and H. Giessen, "Waveguideplasmon polaritons: Strong coupling of photonic and electronic resonances in a metallic photonic crystal slab," Phys. Rev. Lett. 91, 183901 (2003); e-print arXiv:1011.1669v3.

${ }^{18}$ T. Yoshle, A. Scherer, J. Hendrickson, G. Khitrova, H. M. Gibbs, G. Rupper, C. Ell, O. B. Shchekin, and D. G. Deppe, "Vacuum Rabi splitting with a single quantum dot in a photonic crystal nanocavity," Nature 432, 200-203 (2004).

${ }^{19}$ S. Azzini, D. Gerace, M. Galli, I. Sagnes, R. Braive, A. Lemaître, J. Bloch, and D. Bajoni, "Ultra-low threshold polariton lasing in photonic crystal cavities," Appl. Phys. Lett. 99, 111106 (2011); e-print arXiv:1108.4948.

${ }^{20}$ M. Kaliteevski, I. Iorsh, S. Brand, R. A. Abram, J. M. Chamberlain, A. V. Kavokin, and I. A. Shelykh, "Tamm plasmon-polaritons: Possible electromagnetic states at the interface of a metal and a dielectric Bragg mirror," Phys. Rev. B 76, 165415 (2007).

${ }^{21}$ M. E. Sasin, R. P. Seisyan, M. A. Kalitteevski, S. Brand, R. A. Abram, J. M. Chamberlain, A. Y. Egorov, A. P. Vasil'Ev, V. S. Mikhrin, and A. V. Kavokin, "Tamm plasmon polaritons: Slow and spatially compact light," Appl. Phys. Lett. 92, 251112 (2008).

${ }^{22}$ O. Gazzano, S. M. De Vasconcellos, K. Gauthron, C. Symonds, J. Bloch, P. Voisin, J. Bellessa, A. Lemaître, and P. Senellart, "Evidence for confined Tamm plasmon modes under metallic microdisks and application to the control of spontaneous optical emission," Phys. Rev. Lett. 107, 247402 (2011); e-print arXiv:1106.4437v2.

${ }^{23}$ C. Symonds, G. Lheureux, J. P. Hugonin, J. J. Greffet, J. Laverdant, G. Brucoli, A. Lemaitre, P. Senellart, and J. Bellessa, "Confined Tamm plasmon lasers," Nano Lett. 13, 3179-3184 (2013).

${ }^{24}$ T. Braun, V. Baumann, O. Iff, S. Höfling, C. Schneider, and M. Kamp, "Enhanced single photon emission from positioned InP/GaInP quantum dots coupled to a confined Tamm-plasmon mode," Appl. Phys. Lett. 106, 041113 (2015); e-print arXiv:1501.0745.

${ }^{25}$ C. Symonds, S. Azzini, G. Lheureux, A. Piednoir, J. Benoit, A. Lemaitre, P. Senellart, and J. Bellessa, "High quality factor confined Tamm modes," Sci. Rep. 7, 3859 (2017).

${ }^{26}$ G. Lheureux, S. Azzini, C. Symonds, P. Senellart, A. Lemaître, C. Sauvan, J. P. Hugonin, J. J. Greffet, and J. Bellessa, "Polarization-controlled confined Tamm plasmon lasers," ACS Photonics 2, 842-848 (2015).

${ }^{27}$ R. Brückner, A. A. Zakhidov, R. Scholz, M. Sudzius, S. I. Hintschich, H. Früb, V. G. Lyssenko, and K. Leo, "Phase-locked coherent modes in a patterned metalorganic microcavity," Nat. Photonics 6, 322-326 (2012).

${ }^{28}$ H. S. Nguyen, F. Dubois, T. Deschamps, S. Cueff, A. Pardon, J. L. Leclercq, C. Seassal, X. Letartre, and P. Viktorovitch, "Symmetry breaking in photonic crystals: On-demand dispersion from flatband to Dirac cones," Phys. Rev. Lett. 120, 066102 (2018); e-print arXiv:1711.07588.

${ }^{29}$ S. Combrié, P. Colman, A. De Rossi, T. Mei et al., "Vertical high emission in photonic crystal nanocavities by band-folding design," Phys. Rev. B 82, 075120 (2010).

${ }^{30} \mathrm{C}$. Vandenbem, "Electromagnetic surface waves of multilayer stacks: Coupling between guided modes and Bloch modes," Opt. Lett. 33, 2260-2262 (2008).

${ }^{31}$ M. C. F. Dobbelaar, S. Greveling, and D. van Oosten, "Large area photonic crystal cavities: A local density approach," Opt. Express 23, 7481 (2015).

${ }^{32}$ X. Huang, Y. Lai, Z. H. Hang, H. Zheng, and C. T. Chan, "Dirac cones induced by accidental degeneracy in photonic crystals and zero-refractive-index materials," Nat. Mater. 10, 582-586 (2011).

${ }^{33}$ Y. Li, S. Kita, P. Muñoz, O. Reshef, D. I. Vulis, M. Yin, M. Lončar, and E. Mazur, "On-chip zero-index metamaterials," Nat. Photonics 9, 738-742 (2015).

${ }^{34}$ B. Zhen, C. W. Hsu, Y. Igarashi, L. Lu, I. Kaminer, A. Pick, S.-L. Chua, J. D. Joannopoulos, and M. Soljačić, "Spawning rings of exceptional points out of Dirac cones," Nature 525, 354-358 (2015).

${ }^{35}$ R. A. Vicencio, C. Cantillano, L. Morales-Inostroza, B. Real, C. Mejía-Cortés, S. Weimann, A. Szameit, and M. I. Molina, "Observation of localized states in Lieb photonic lattices,” Phys. Rev. Lett. 114, 245503 (2015).

${ }^{36}$ S. Mukherjee, A. Spracklen, D. Choudhury, N. Goldman, P. Öhberg, E. Andersson, and R. R. Thomson, "Observation of a localized flat-band state in a photonic Lieb lattice,” Phys. Rev. Lett. 114, 245504 (2015); e-print arXiv:1412.6342.

${ }^{37}$ L. Lu, J. D. Joannopoulos, and M. Soljačić, "Topological photonics," Nat. Photonics 8, 821-829 (2014); e-print arXiv:1408.6730. 
${ }^{38}$ V. M. Shalaev, “Optical negative-index metamaterials," Nat. Photonics 1, 41-48 (2007).

${ }^{39}$ N. Segal, S. Keren-Zur, N. Hendler, and T. Ellenbogen, "Controlling light with metamaterial-based nonlinear photonic crystals," Nat. Photonics 9, 180-184 (2015).

${ }^{40}$ F. Xia, H. Wang, D. Xiao, M. Dubey, and A. Ramasubramaniam, "Twodimensional material nanophotonics," Nat. Photonics 8, 899-907 (2014); e-print arXiv:1410.3882.

${ }^{41}$ Y. J. Noori, Y. Cao, J. Roberts, C. Woodhead, R. Bernardo-Gavito, P. Tovee, and R. J. Young, "Photonic crystals for enhanced light extraction from 2D materials," ACS Photonics 3, 2515-2520 (2016); e-print arXiv:1607.04973.

${ }^{42}$ L. Feng, R. El-Ganainy, and L. Ge, "Non-Hermitian photonics based on paritytime symmetry," Nat. Photonics 11, 752-762 (2017).

${ }^{43}$ R. El-Ganainy, K. G. Makris, M. Khajavikhan, Z. H. Musslimani, S. Rotter, and D. N. Christodoulides, "Non-Hermitian physics and PT symmetry," Nat. Phys. 14, 11-19 (2018)

${ }^{44}$ N. Le Thomas, R. Houdré, M. V. Kotlyar, D. O’Brien, and T. F. Krauss, "Exploring light propagating in photonic crystals with Fourier optics," J. Opt. Soc. Am. B 24, 2964-2971 (2007) 